\title{
Trepostome bryozoans buck the trend and ignore calcite-aragonite seas
}

\author{
Marcus M. Key Jr ${ }^{1}$ (D) Patrick N. Wyse Jackson ${ }^{2} \cdot$ Catherine M. Reid $^{3}$
}

Received: 28 April 2021 / Revised: 11 June 2021 / Accepted: 13 July 2021 / Published online: 9 October 2021

(C) The Author(s) 2021

\begin{abstract}
Trepostome bryozoan skeletalisation did not passively respond to changes in seawater chemistry associated with calcitearagonite seas. According to Stanley and others, trepostome bryozoans were passive hypercalcifiers. However, if this was the case, we would expect their degree of calcitic colony calcification to have decreased across the Calcite I Sea to the Aragonite II Sea at its transition in the Middle Mississippian. Data from the type species of all 184 trepostome genera from the Early Ordovician to the Late Triassic were utilised to calculate the Bryozoan Skeletal Index (BSI) as a proxy for the degree of calcification. BSI values and genus-level diversity did not decrease across the transition from the Calcite I Sea to the Aragonite II Sea. Nor were there any changes in the number of genus originations and extinctions. This suggests that trepostome bryozoans were not passive hypercalcifiers but active biomineralisers that controlled the mineralogy and robustness of their skeletons regardless of changes in seawater chemistry.
\end{abstract}

Keywords Trepostomata $\cdot$ Bryozoa $\cdot$ Skeletalisation $\cdot$ Calcite-aragonite seas

\section{Introduction}

Stanley and Hardie $(1998,1999)$ synthesised a theory of temporal geochemical variation in the oceans through the Phanerozoic (last 550 Myr), describing oscillations in the mineralogy of marine inorganic and biogenic carbonates, with intervals dominated by aragonite and/or high-Mg calcite (aragonite seas) and low-Mg calcite (calcite seas) (Sandberg 1975, 1983; Hardie 1996). They argued that temporal variation in the $\mathrm{Mg} / \mathrm{Ca}$ ratio of seawater was driven by changes in plate tectonic spreading rates at mid-ocean ridges. Faster seafloor spreading lowers the $\mathrm{Mg} / \mathrm{Ca}$ ratio in the oceans which favours

Patrick N. Wyse Jackson

wysjcknp@tcd.ie

Marcus M. Key, Jr

key@dickinson.edu

1 Department of Earth Sciences, Dickinson College, Carlisle, Pennsylvania 17013-2896, USA

2 Patrick N. Wyse Jackson, Department of Geology, Trinity College, Dublin 2, Ireland

3 Catherine M. Reid, School of Earth and Environment, University of Canterbury, Christchurch 8140, New Zealand biomineralisation of low-Mg calcite. The opposite creates aragonite conditions.

Their supporting data were derived from marine cements, ooids, evaporites, and tropical hypercalcifying organisms (e.g. calcareous green algae, sponges, and corals). This work has been subsequently supported by studies on coralline algae (Stanley et al. 2002), rugose corals (Webb and Sorauf 2002), crinoids (Dickson 2002, 2004), echinoids, crabs, shrimps, and serpulid worms (Ries 2004), as well as coccoliths (Stanley et al. 2005). This secular carbonate mineralogy model is also supported by oscillations in the composition of fluid inclusions in marine evaporites (Lowenstein et al. 2001), the $\mathrm{Sr}: \mathrm{Ca}$ ratio of biogenic carbonate (Steuber and Veizer 2002), and bromine concentrations in halite (Siemann 2003). In contrast, others have argued that the secular pattern in carbonate mineralogy is controlled by oscillations in atmospheric $p \mathrm{CO}_{2}$ (Mackenzie and Pigott 1981; Sandberg 1983; Wilkinson and Givens 1986; Burton and Walter 1991; Zhuravlev and Wood 2009). The debate over the driving mechanism continues (Montañez 2002; Schlager 2005; Ries 2010; van Dijk et al. 2016; Turchyn and DePaolo 2019).

Regardless of the causal process, if seawater chemistry changes are global in scope, then it might be expected that geochemical signals should be expressed beyond Stanley and Hardie's largely tropical examples and beyond these few 
biogenic carbonate producers. If bryozoans are passive hypercalcifying animals, they could provide an independent test of these hypothesised temporal variations in seawater chemistry. Bryozoans are mineralogically variable (Smith et al. 1998). They have an excellent fossil record from the Ordovician to the present (Taylor 1993; Ernst 2020). They have adapted to a cool-water temperate to polar palaeolatitudinal distribution through the Phanerozoic (Taylor and Allison 1998). As colonial animals, they have at times been important hypercalcifying carbonate producers (Stanley and Hardie 1998, 1999; Stanley 2006).

Stanley and Hardie (1998, 1999), Stanley et al. (2002), and Montañez (2002) argue that seawater chemistry exerts a strong control on the biomineralogy of morphologically simple clades that have weak control over their calcification. In addition to algae, sponges, and corals, they explicitly mention bryozoans as an example. Stanley and Hardie $(1998,1999)$ and Stanley (2006) suggest the fossil abundance and degree of calcification of 'stony' stenolaemate bryozoans (i.e. trepostomes) were controlled by seawater chemistry because they were considered to be passive hypercalcifiers.

All trepostomes were calcitic (Taylor et al. 2010; Taylor 2020). While the detailed mineralogy of most trepostomes remains unknown, of those whose mineralogy has been determined, all were low-Mg calcite (LMC) with the exception of one, Nicholsonella from the Ordovician of North America, which was high-Mg calcite (Tavener-Smith and Williams 1972; Taylor and Wilson 1999; Smith et al. 2006). Assuming all trepostomes were LMC, we would predict their degree of calcification to be higher during the Calcite I Sea than the Aragonite II sea. If they are not significantly different, that suggests that bryozoans are able to actively biomineralise even in adverse conditions, thus are not passive as suggested by Stanley and others (Stanley and Hardie 1998, 1999; Stanley 2006). The fossil record of trepostomes overlaps with the Calcite I Sea-Aragonite II Sea transition which occurred in the Middle Mississippian of the Early Carboniferous. We will test the hypothesis that trepostomes were passive hypercalcifying animals by quantifying their degree of calcification across this transition.

\section{Materials and methods}

Trepostomes are an order of stenolaemate bryozoans which evolved in the early Ordovician and went extinct in the Late Triassic (Taylor 2020). Most abundant in the early Palaeozoic, they are informally known as 'stony bryozoans'. All species of trepostomes have long, tubular zooids. Although encrusting, massive, frondose, and bifoliate colonies occur, most are ramose with bifurcating cylindrical branches that form small bush-like colonies (Key et al. 2016). These branches have an inner thinner-walled endozone surrounded by a peripheral thicker-walled exozone (Fig. 1).

We used the Bryozoan Skeletal Index (BSI) developed by Wyse Jackson et al. (2020) as a proxy for the degree of calcification of the trepostomes. The BSI measures the relative proportion of skeletal carbonate to intrazoarial void space in stenolaemate bryozoan colonies. It is calculated from exozone width in longitudinal or transverse section (EW), zooecial wall thickness between adjacent autozooecial apertures in shallow

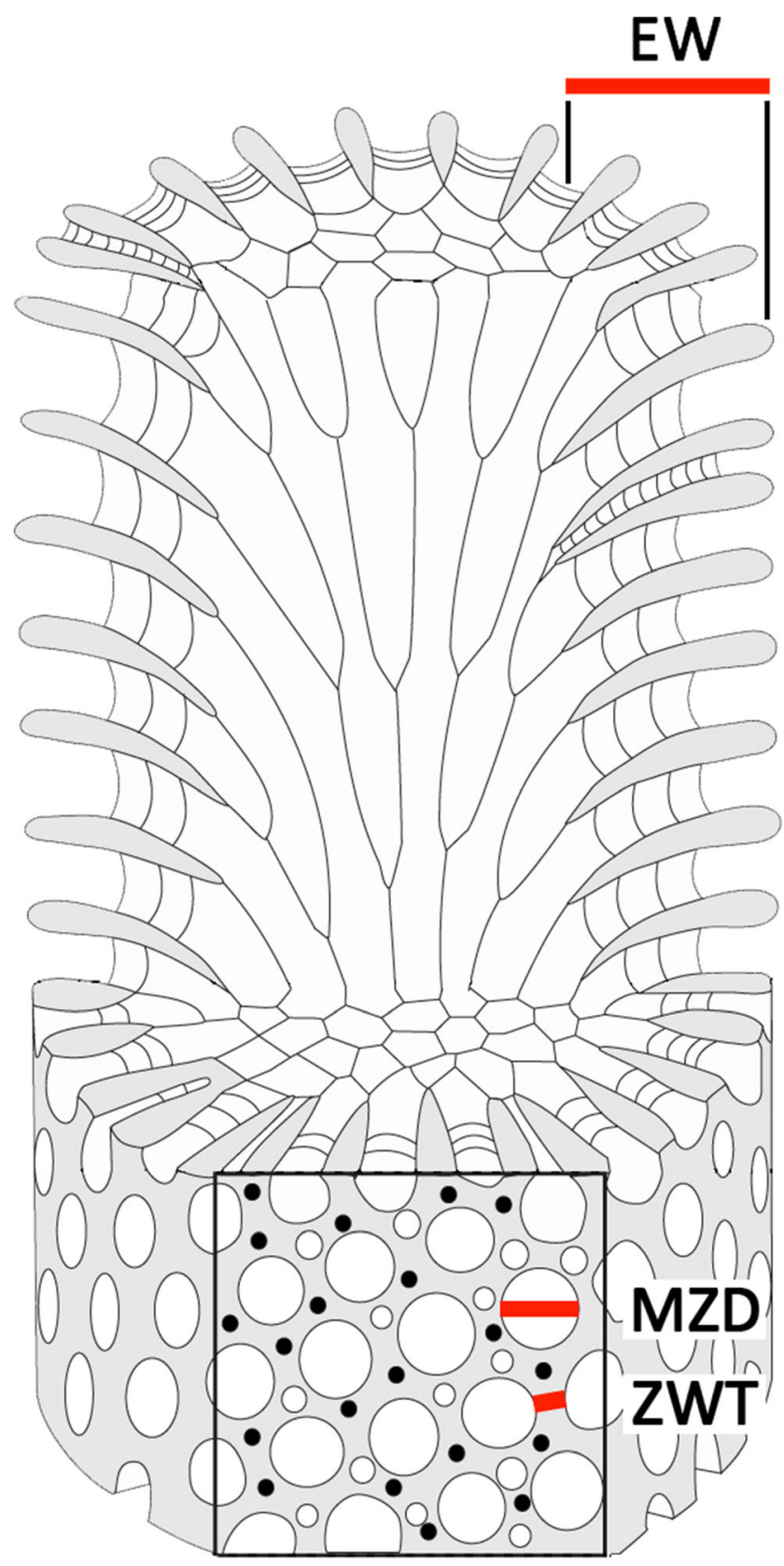

Fig. 1 Morphological characters used to compute the Bryozoan Skeletal Index (BSI). From Wyse Jackson et al. (2020, fig. 2). Abbreviations: EW, exozone width; MZD, autozooecial aperture diameter; ZWT, zooecial wall thickness 
tangential section (ZWT), and autozooecial aperture diameter in shallow tangential section (MZD) using the following equation: $\mathrm{BSI}=((\mathrm{EW} \times \mathrm{ZWT}) / \mathrm{MZD}) \times 100$ (Fig. 1). The multiplication factor of $\times 100$ ensures that BSI is a whole number. Lower numbers represent lower levels of calcification and higher numbers the converse. For circular autozooecial apertures, the maximum MZD was measured. For oval-shaped apertures, both maximum and minimum MZD were determined and the average of the means of these used in the equation. For multilaminar encrusting species in which successive layers overgrow those immediately beneath, the thickness of the exozone in one lamina was measured.

We calculated BSI for the type species of each trepostome genus included in the forthcoming chapter on Order Trepostomata for the Treatise on Invertebrate Paleontology, Part G, Bryozoa, Revised, Volume 2 (Boardman and Buttler, forthcoming). We included all genera, regardless of their zoarial habit (Wyse Jackson et al. 2020, fig. 1). We determined the zoarial habit (i.e. ramose, encrusting, massive, foliose, bifoliate, or mixed) for each type species. When possible, we determined the zoarial habit from the type species' original publication and plates. For many species erected in the nineteenth century for which illustrations are either lacking or uninformative, we used the descriptions from the forthcoming Treatise on Invertebrate Paleontology's trepostome volume (Boardman and Buttler, forthcoming). Our reliance on type specimens of type species of each genus assumes they are representative of the genus. They should be, as that is the fundamental purpose of type specimens and type species (ICZN 1999). But this approach requires the assumption that there is no significant intrageneric, intraspecific, or intracolony variation which is known to exist in bryozoans (Taylor 2020).

When possible, we acquired mean values for the three BSI morphometric parameters (EW, ZWT, MZD) from the type specimens of the type species of each genus. For more recently erected species, the morphometric data were extracted from published data tables. For older species descriptions lacking data tables, we used data from recent revisions of the taxa. Where means or ranges were not reported in the literature, replicate measurements for each parameter were taken directly from figured material accompanying the type descriptions or from the plates of Boardman and Buttler (forthcoming).

In some taxa, the dimensions of some parameters could not be determined from the type specimens of the type species. In these rare cases, it was measured from non-type material from the same species or from another species whose stratigraphic age and geographical location matched as closely as possible to those of the type species. This was mainly necessary for some taxa forming encrusting and massive zoaria in which the exozone width could not be accurately measured. In all such cases, the other parameter dimensions were compared with those of the type species to ensure that they were equivalent.
Type species ages were defined as the midpoint of the type locality stratigraphic age ranges from Shanan Peters' Macrostrat.org. Where type locality stratigraphic ages were not included in Macrostrat.org, we used updated stratigraphic concepts revised since the description of the species or local literature for some non-North American species. Genus stratigraphic ranges were based on the genus descriptions from Boardman and Buttler (forthcoming) and augmented with Phil Bock's Bryozoa.net. All numerical ages were based on the latest version of the ICS geologic time scale (Cohen et al. 2020).

We followed the methodology of Kiessling et al. (2008) and van Dijk et al. (2016) in using $t$ tests to analyse for significant difference between the mean values before and after the Calcite I-Aragonite II transition. We used two approaches. The first examined BSI values across this transition using the midpoint of the age of the type species of each genus. The second used the stratigraphic range of each genus to look for a drop in the diversity of trepostomes across the transition.

Hardie (1996) put the Calcite I-Aragonite II transition date at $335 \mathrm{Ma}$ based on the 1983 DNAG geologic time scale (Palmer 1983). Porter (2010, fig. 3), using a more recent geologic time scale (i.e. the 2008 ICS version whose Carboniferous boundary ages have not changed since then; Cohen et al. 2020), showed the transition date at $342 \mathrm{Ma}$. According to van Dijk et al. (2016), there is some disagreement about when the Calcite I-Aragonite II transition occurred. Depending on if one uses Stanley and Hardie's $(1998,1999) \mathrm{Mg} / \mathrm{Ca}$ model or Farkaš et al.'s (2007) $\mathrm{Mg} / \mathrm{Ca}$ model, the estimated timing of transition ranges from 333 to $350 \mathrm{Ma}$ (van Dijk et al. 2016, fig. 1). In contrast, Porter (2010, fig. 3) and Balthasar and Cusack (2015, fig. 2) suggest there is no period of uncertainty at this transition as opposed to the other transitions. Balthasar and Cusack (2015, fig. 2) and Quattrini et al. (2020, fig. 2) place it at $350 \mathrm{Ma}$. Therefore, we chose to test for changes to trepostome BSI across the transition at all three proposed ages of 333, 342, and $350 \mathrm{Ma}$.

\section{Results}

This study included all 184 trepostome genera (Table 1). They ranged from Orbiramus from the Tremadocian stage of the Early Ordovician of China to Styloclema from the Norian stage of the Late Triassic of New Zealand. That is an expanse of $265 \mathrm{Myr}$ from 480 to $215 \mathrm{Ma}$ (Fig. 2). The type species' BSI values ranged from 3 to 211 (mean: 44; standard deviation: 37) (Table 1). As determined by two sample $t$ tests, there is no significant (i.e. $P>0.05$ ) change in BSI values from before to after the 350 Ma mark, 342 Ma mark, or $333 \mathrm{Ma}$ mark (Table 2). The mean BSI of all genera per 1 Myr bins across the ranges of all genera varied from 29 to 95 (mean: 53; 


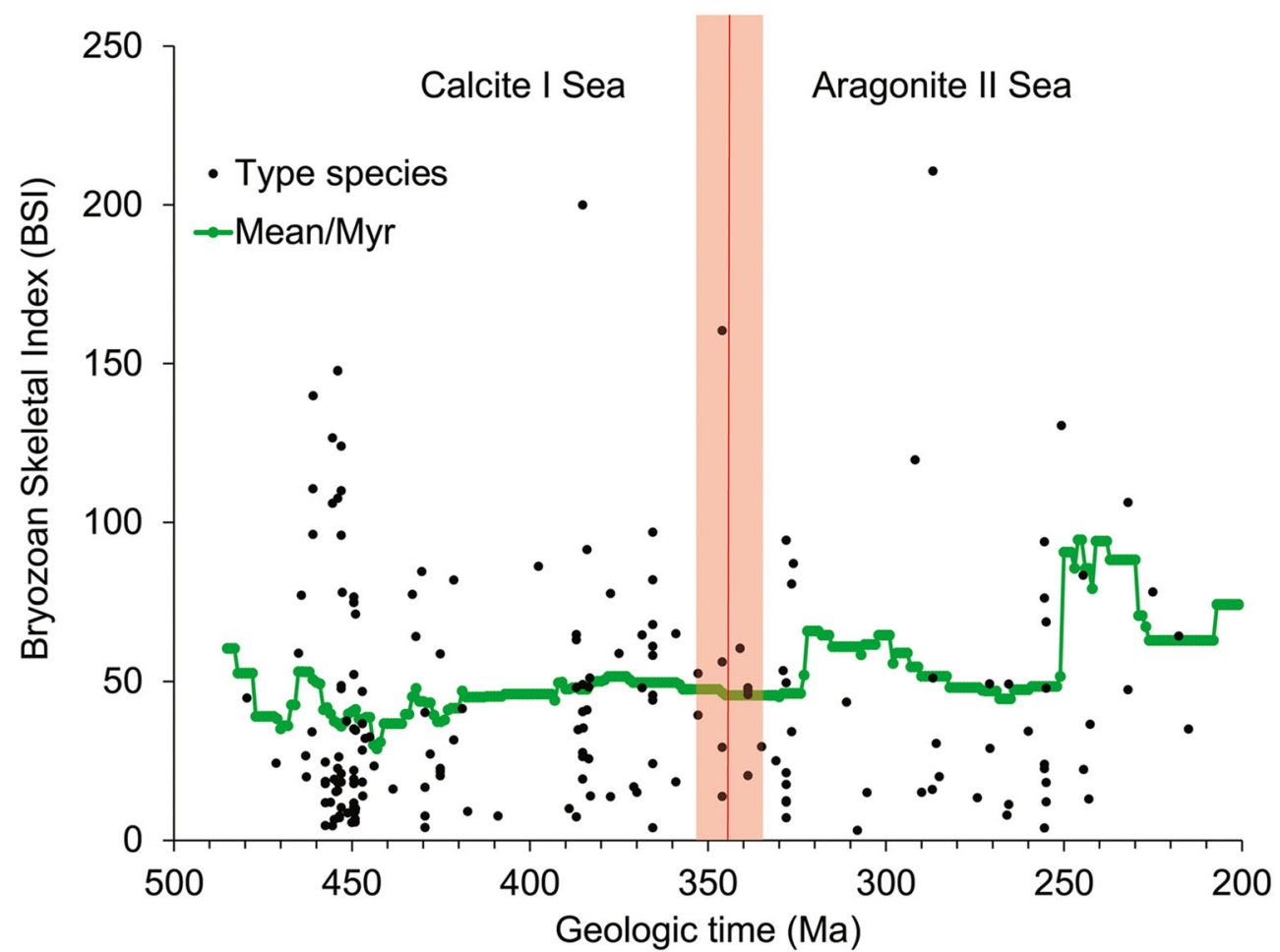

Fig. 2 Trepostome Bryozoan Skeletal Index (BSI) over geologic time based on each genus' type species mean age and the mean BSI per million years. Three different proposed ages for the transition from the
Calcite I Sea to the Aragonite II Sea are shown by the shaded red box. Left edge of shaded box is $350 \mathrm{Ma}$, vertical line in centre is $342 \mathrm{Ma}$, and right edge of shaded box is $333 \mathrm{Ma}$ standard deviation: 14) (Fig. 2). There is essentially no change in mean BSI values at either the 350 Ma mark ( 47.5 before and after), $342 \mathrm{Ma}$ mark (45.6 before and after), or $333 \mathrm{Ma}$ (45.6 before and after).

Does the lack of change in BSI values hold up for individual zoarial types? There are not enough foliose and bifoliate trepostomes $(n=6)$, so they were excluded from this additional analysis. Genera whose type species are ramose or most commonly ramose $(n=125)$ showed no significant change in BSI across the Calcite I Sea/Aragonite II Sea transition, regardless of which of the three transition ages were chosen (two sample ttests, $P>0.05$, Table 2 ). Genera whose type species are encrusting or most commonly encrusting $(\mathrm{n}=38)$ showed no significant change in BSI across the Calcite I Sea/Aragonite II Sea transition, regardless of which of the three transition ages were chosen (two sample $t$ tests, $P>0.05$, Table 2). Finally, genera whose type species are massive $(n=15)$ showed no significant change in BSI across the Calcite I Sea/Aragonite II Sea transition, regardless of which of the three transition ages were chosen (two sample $t$ tests, $P>0.05$, Table 2). Therefore, the pattern of lack of change in degree of calcification in response to changes in seawater chemistry is robust across each trepostome zoarial habit. Additionally, there is no change in genus-level trepostome diversity, originations, or extinctions across this transition (Fig. 3).

\section{Discussion and conclusions}

There was no significant decrease in BSI values as the calcitic trepostomes transitioned from the Calcite I Sea into the Aragonite II Sea (Table 2, Fig. 2). There was no change in diversity at either the 350 Ma mark ( 27 genera before and after), 342 Ma mark (26 genera before and after), or $333 \mathrm{Ma}$ (26 genera before and after) (Fig. 3). In addition to the diversity graph being relatively flat during the transition, there was essentially no change in origination or extinction rate (Fig. 3). If trepostomes were responding to the changing ocean chemistry structurally, then one would expect new taxa to appear as trepostome systematics is based on skeletal structures. Trepostomes were structurally resilient to whatever was changing in seawater at the transition. If trepostomes were passive hypercalcifiers, then one would expect a drop in BSI values as the calcitic trepostomes transitioned from the Calcite I Sea into the Aragonite II Sea. If trepostomes were passive hypercalcifiers, then one could expect a macroevolutionary drop in diversity as the calcitic trepostomes transitioned from the Calcite I Sea into the Aragonite II Sea.

In contrast, two prominent diversity drops are visible in Fig. 3 and mark the mass extinctions defining the Ordovician-Silurian boundary (444 Ma; Sheehan 2001; Bond and Gasby 2020) and Permian-Triassic boundary (251 
Table 1 Trepostome genera Bryozoan Skeletal Index (BSI) values, type species ages, and genera age ranges. Arranged by type species age.

\begin{tabular}{|c|c|c|c|c|c|}
\hline Genus & Type species & $\begin{array}{l}\text { Bryozoan Skeletal } \\
\text { Index (BSI) }\end{array}$ & $\begin{array}{l}\text { Type species } \\
\text { age (Ma) }\end{array}$ & $\begin{array}{l}\text { Lower range } \\
\text { of genus (Ma) }\end{array}$ & $\begin{array}{l}\text { Upper range } \\
\text { of genus (Ma) }\end{array}$ \\
\hline Styloclema & morozovae & 35.0 & 215.0 & 227.0 & 208.5 \\
\hline Buria & improvisa & 64.3 & 217.8 & 227.0 & 208.5 \\
\hline Metastenodiscus & zealandicus & 78.1 & 225.0 & 227.0 & 201.3 \\
\hline Dyscritellopsis & isoseptatus & 47.4 & 232.0 & 237.0 & 201.3 \\
\hline Zozariella & stellata & 106.3 & 232.0 & 247.2 & 227.0 \\
\hline Phragmotrypa & ordinata & 36.5 & 242.7 & 247.2 & 242.0 \\
\hline Reptonoditrypa & cautica & 12.9 & 243.0 & 244.0 & 242.0 \\
\hline Tebitopora & orientalis & 22.2 & 244.5 & 242.0 & 208.5 \\
\hline Vysokella & acanthostylica & 83.4 & 244.6 & 247.2 & 242.0 \\
\hline Arcticopora & christiei & 130.6 & 250.7 & 251.9 & 201.3 \\
\hline Iraidina & damperovi & 47.9 & 255.0 & 259.1 & 251.9 \\
\hline Neoeridocampylus & rarus & 68.8 & 255.0 & 259.1 & 251.9 \\
\hline Parastenodiscus & nevolinae & 12.0 & 255.0 & 358.9 & 251.9 \\
\hline Ruzhencevia & elegans & 18.1 & 255.0 & 273.0 & 251.9 \\
\hline Anisotrypella & borealis & 76.2 & 255.5 & 259.1 & 251.9 \\
\hline Maychella & tuberculata & 3.8 & 255.5 & 298.9 & 251.9 \\
\hline Permolioclema & iraidae & 23.9 & 255.5 & 259.1 & 251.9 \\
\hline Permopora & prima & 93.9 & 255.5 & 273.0 & 251.9 \\
\hline Ulrichotrypella & kapitzae & 22.5 & 255.5 & 268.8 & 251.9 \\
\hline Araxopora & spinigera var. araxensis & 34.3 & 260.0 & 298.9 & 251.9 \\
\hline Autospinifera & rossae & 49.2 & 265.5 & 268.8 & 265.1 \\
\hline Stellahexaformis & gersterensis & 11.2 & 265.5 & 273.0 & 251.9 \\
\hline Utgaardostylus & stylata & 7.8 & 266.0 & 268.8 & 265.1 \\
\hline Neoeridotrypella & pulchra & 28.9 & 270.8 & 290.1 & 251.9 \\
\hline Dyscritellina & clivosa & 49.2 & 270.9 & 293.5 & 259.1 \\
\hline Crockfordia & multinodata & 13.3 & 274.3 & 290.1 & 265.1 \\
\hline Toulapora & svalbardensis & 20.0 & 285.0 & 293.5 & 283.5 \\
\hline Ulrichotrypa & ramulosa & 30.4 & 285.9 & 298.9 & 251.9 \\
\hline Nansenopora & peculiaris & 51.1 & 286.8 & 298.9 & 273.0 \\
\hline Stenodiscus & moniliformis & 210.7 & 286.8 & 323.2 & 230.0 \\
\hline Paramaychellina & spinosa & 16.0 & 287.0 & 298.9 & 251.9 \\
\hline Hinganella & laeviuscula & 15.0 & 290.0 & 293.5 & 251.9 \\
\hline Stenopora & tasmaniensis & 119.8 & 291.8 & 358.9 & 251.9 \\
\hline Crustoporella & alekseevi & 14.9 & 305.4 & 307.0 & 303.7 \\
\hline Mishulgella & stellata & 3.0 & 308.0 & 315.2 & 307.0 \\
\hline Rhombotrypella & astragaloides & 43.5 & 311.1 & 323.2 & 251.9 \\
\hline Dunaevella & shishovae & 87.1 & 326.0 & 330.9 & 319.0 \\
\hline Anisotrypa & spinulosa & 80.6 & 326.5 & 427.4 & 323.2 \\
\hline Pseudobatostomella & symmetrica & 34.1 & 326.5 & 419.2 & 201.3 \\
\hline Callocladia & romingeri & 17.5 & 328.0 & 443.8 & 298.9 \\
\hline Coeloclemis & tumida & 21.2 & 328.0 & 358.9 & 251.9 \\
\hline Dyscritella & insigne & 94.4 & 328.0 & 419.2 & 201.3 \\
\hline Idioclema & regularis & 49.6 & 328.0 & 358.9 & 323.2 \\
\hline Pycnopora & frondosa & 7.0 & 328.0 & 358.9 & 323.2 \\
\hline Stenocladia & robusta & 12.4 & 328.0 & 358.9 & 323.2 \\
\hline Stenoporella & elegans & 11.9 & 328.0 & 330.9 & 323.2 \\
\hline Maychellina & aliena & 53.4 & 328.9 & 358.9 & 251.9 \\
\hline Astralochoma & helenae & 25.0 & 330.9 & 330.9 & 323.2 \\
\hline Nipponostenopora & elegantula & 29.4 & 334.9 & 358.9 & 298.9 \\
\hline Hinaclema & hinaensis & 45.9 & 338.8 & 358.9 & 283.5 \\
\hline Hunanopora & lobatum & 48.0 & 338.8 & 358.9 & 323.2 \\
\hline Stenophragmidium & sinensis & 20.3 & 338.8 & 346.7 & 330.9 \\
\hline Triznotrypa & subtomiensis & 47.3 & 338.8 & 387.7 & 330.9 \\
\hline Leioclema & concentrica & 60.4 & 341.0 & 485.4 & 201.3 \\
\hline Nikiforopora & punctata & 60.5 & 341.0 & 358.9 & 298.9 \\
\hline Cyclopora & fungia & 29.2 & 346.0 & 358.9 & 323.2 \\
\hline Proutella & discoidea & 13.7 & 346.0 & 358.9 & 323.2 \\
\hline Tabulipora & nalvkini & 56.2 & 346.0 & 419.0 & 251.9 \\
\hline Tabuliporella & urii & 160.4 & 346.0 & 358.9 & 283.5 \\
\hline Raissiella & bystrensis & 52.5 & 352.8 & 372.2 & 346.7 \\
\hline Volnovachia & distincta & 39.4 & 352.8 & 358.9 & 323.2 \\
\hline Aisenvergia & cylindrica & 65.1 & 359.0 & 358.9 & 346.7 \\
\hline Crustopora & tuberculata & 18.3 & 359.0 & 407.6 & 323.2 \\
\hline Armillopora & sinensis & 61.1 & 365.5 & 372.2 & 358.9 \\
\hline
\end{tabular}


Table 1 (continued)

\begin{tabular}{|c|c|c|c|c|c|}
\hline Genus & Type species & $\begin{array}{l}\text { Bryozoan Skeletal } \\
\text { Index (BSI) }\end{array}$ & $\begin{array}{l}\text { Type species } \\
\text { age (Ma) }\end{array}$ & $\begin{array}{l}\text { Lower range } \\
\text { of genus }(\mathrm{Ma})\end{array}$ & $\begin{array}{l}\text { Upper range } \\
\text { of genus (Ma) }\end{array}$ \\
\hline Eodyscritella & clathrata & 45.6 & 365.5 & 372.2 & 358.9 \\
\hline Fitzroyopora & multiseptatum & 58.2 & 365.5 & 382.7 & 358.9 \\
\hline Granivallum & shaodongensis & 68.0 & 365.5 & 382.7 & 358.9 \\
\hline Multiphragma & hunanensis & 81.9 & 365.5 & 372.2 & 358.9 \\
\hline Polyspinopora & granulosa & 96.9 & 365.5 & 372.2 & 358.9 \\
\hline Schulgina & oscarensis & 44.1 & 365.5 & 382.7 & 358.9 \\
\hline Sinoatactotoechus & fistulosum & 24.1 & 365.5 & 372.2 & 358.9 \\
\hline Triplopora & nesterenkoae & 3.8 & 365.5 & 372.2 & 358.9 \\
\hline Hyalotoechus & duncani & 48.0 & 368.5 & 379.0 & 358.0 \\
\hline Linotaxis & magna & 64.7 & 368.5 & 379.0 & 358.0 \\
\hline Zefrehopora & asynithis & 15.0 & 370.0 & 372.2 & 358.9 \\
\hline Percyopora & tubulata & 16.7 & 370.8 & 382.7 & 358.9 \\
\hline Petalotrypa & compressa & 58.9 & 375.0 & 393.3 & 382.7 \\
\hline Amplexoporella & ornamentata & 77.6 & 377.4 & 382.7 & 372.2 \\
\hline Paralioclema & ninae & 13.7 & 377.4 & 419.2 & 247.2 \\
\hline Atactotoechus & typicus & 13.8 & 383.0 & 419.2 & 358.9 \\
\hline Kysylschinipora & nekhoroschevi & 51.1 & 383.2 & 387.7 & 358.9 \\
\hline Dyoidophragma & typicus & 25.6 & 383.5 & 393.3 & 358.9 \\
\hline Stereotoechus & typicale & 48.2 & 383.5 & 391.0 & 379.0 \\
\hline Eostenopora & picta & 91.4 & 384.0 & 388.0 & 379.0 \\
\hline Leptotrypella & barrandi & 41.0 & 384.0 & 391.0 & 379.0 \\
\hline Eridocampylus & ulrichi & 26.7 & 385.0 & 388.0 & 379.0 \\
\hline Microcampylus & typicus & 35.3 & 385.0 & 388.0 & 379.0 \\
\hline Abakana & macrospina & 49.0 & 385.2 & 387.7 & 382.7 \\
\hline Boardmanella & richardi & 27.6 & 385.2 & 427.4 & 387.7 \\
\hline Diphragmoides & paradoxus & 19.2 & 385.2 & 390.0 & 384.0 \\
\hline Eifelipora & ramosa & 26.3 & 385.2 & 390.0 & 384.0 \\
\hline Minussina & maculosa & 200.0 & 385.2 & 419.2 & 358.9 \\
\hline Neotrematopora & typica & 40.4 & 385.2 & 427.4 & 330.0 \\
\hline Eridotrypella & obliqua & 34.8 & 386.5 & 391.0 & 379.0 \\
\hline Loxophragma & lechrium & 64.8 & 387.0 & 391.0 & 382.0 \\
\hline Polycylindricus & amphelicta & 48.0 & 387.0 & 419.2 & 358.9 \\
\hline Pycnobasis & asphinctus & 63.1 & 387.0 & 391.0 & 382.0 \\
\hline Trachytoechus & typicus & 7.3 & 387.0 & 427.4 & 358.0 \\
\hline Multihemiphragma & tenuis & 9.9 & 389.0 & 393.2 & 382.7 \\
\hline Mongoloclema & ignotum & 86.2 & 397.6 & 407.6 & 387.7 \\
\hline Koneprusiella & armata & 7.6 & 409.0 & 419.2 & 393.3 \\
\hline Badoglioporina & parvulipora & 9.0 & 417.5 & 419.2 & 393.3 \\
\hline Diplostenopora & siluriana & 41.4 & 419.0 & 422.0 & 413.0 \\
\hline Astroviella & mukhovetskensis & 31.5 & 421.4 & 427.4 & 393.3 \\
\hline Astroviellina & porosa & 81.9 & 421.4 & 423.0 & 419.2 \\
\hline Calamotrypa & millichopensis & 20.2 & 425.2 & 427.4 & 423.0 \\
\hline Eridotrypellina & insolens & 58.7 & 425.2 & 427.4 & 423.0 \\
\hline Hemieridotrypa & tsherkesovae & 22.6 & 425.2 & 427.4 & 423.0 \\
\hline Pseudoleptotrypa & podolica & 21.6 & 425.2 & 427.4 & 423.0 \\
\hline Hallopora & elegantula & 27.1 & 428.0 & 458.4 & 393.3 \\
\hline Acanthotrypina & spinosa & 3.9 & 429.5 & 433.4 & 427.4 \\
\hline Badogliopora & ramulosum & 16.6 & 429.5 & 431.0 & 425.0 \\
\hline Leioclemina & tuberculosa & 40.2 & 429.5 & 431.0 & 425.0 \\
\hline Trematopora & spinulifera & 7.6 & 429.5 & 431.0 & 425.0 \\
\hline Diplotrypella & densipora & 84.5 & 430.4 & 433.4 & 427.4 \\
\hline Asperopora & aspera & 64.2 & 432.1 & 433.4 & 427.4 \\
\hline Idiotrypa & parasitica & 77.3 & 433.0 & 431.0 & 425.0 \\
\hline Lioclemella & ohioensis & 16.1 & 438.5 & 443.0 & 436.0 \\
\hline Canavaripora & nitida & 23.4 & 443.8 & 457.0 & 434.0 \\
\hline Acanthotrypella & variabile & 32.5 & 445.0 & 448.0 & 445.2 \\
\hline Jifarahpora & lybiensis & 32.0 & 446.3 & 449.7 & 443.0 \\
\hline Rhombotrypa & quadrata & 13.9 & 447.0 & 448.0 & 445.2 \\
\hline Anaphragma & mirabile & 46.8 & 447.1 & 448.0 & 445.2 \\
\hline Calloporella & harrisi & 28.3 & 447.1 & 448.0 & 445.2 \\
\hline Gortanipora & crenulata & 18.3 & 447.1 & 456.0 & 445.2 \\
\hline Stigmatella & bassleri & 36.7 & 447.1 & 451.0 & 445.2 \\
\hline Batostomella & gracilis & 71.3 & 449.0 & 451.0 & 445.2 \\
\hline Leptotrypa & ramosa & 9.9 & 449.0 & 458.4 & 443.8 \\
\hline
\end{tabular}


Table 1 (continued)

\begin{tabular}{|c|c|c|c|c|c|}
\hline Genus & Type species & $\begin{array}{l}\text { Bryozoan Skeletal } \\
\text { Index (BSI) }\end{array}$ & $\begin{array}{l}\text { Type species } \\
\text { age (Ma) }\end{array}$ & $\begin{array}{l}\text { Lower range } \\
\text { of genus (Ma) }\end{array}$ & $\begin{array}{l}\text { Upper range } \\
\text { of genus (Ma) }\end{array}$ \\
\hline Parvohallopora & minima & 34.5 & 449.0 & 453.0 & 445.2 \\
\hline Mesotrypa & petechialis & 6.6 & 449.1 & 458.4 & 443.8 \\
\hline Peronopora & decipiens & 9.0 & 449.1 & 457.0 & 442.0 \\
\hline Petigopora & infida & 5.6 & 449.1 & 456.0 & 445.2 \\
\hline Amplexopora & crassimuralis & 21.9 & 449.5 & 459.0 & 445.2 \\
\hline Bodywskites & elegans & 10.4 & 449.5 & 461.2 & 449.2 \\
\hline Dekayia & curvata & 19.2 & 449.5 & 457.0 & 445.2 \\
\hline Discotrypa & mammulata & 11.7 & 449.5 & 451.0 & 447.0 \\
\hline Heterotrypa & aspera & 76.5 & 449.5 & 459.0 & 445.2 \\
\hline Homotrypa & septosa & 52.2 & 449.5 & 456.0 & 445.2 \\
\hline Monticulipora & frondosa & 17.8 & 449.5 & 456.0 & 445.2 \\
\hline Ralfimartites & fistulatus & 35.1 & 449.5 & 471.3 & 449.2 \\
\hline Tetratoechus & lautus & 74.8 & 449.5 & 448.0 & 445.2 \\
\hline Lodenicella & lamellata & 8.8 & 450.0 & 453.0 & 445.2 \\
\hline Bythopora & dendrina & 5.5 & 450.1 & 470.0 & 443.8 \\
\hline Sonninopora & tenuispinosa & 8.5 & 451.2 & 458.4 & 443.8 \\
\hline Polyteichus & novaki & 37.5 & 451.5 & 453.0 & 448.0 \\
\hline Pedrogopora & taylori & 77.9 & 452.7 & 458.4 & 449.3 \\
\hline Balticopora & implicatum & 20.9 & 453.0 & 457.0 & 445.2 \\
\hline Balticoporella & glabrum & 110.1 & 453.0 & 453.5 & 449.5 \\
\hline Batostoma & mutabilis & 124.1 & 453.0 & 451.0 & 445.2 \\
\hline Cyphotrypa & tenuimurale & 10.2 & 453.0 & 458.4 & 443.8 \\
\hline Eridotrypa & typicalis & 47.7 & 453.0 & 456.0 & 430.0 \\
\hline Monotrypa & acervulosa & 18.3 & 453.0 & 458.4 & 443.8 \\
\hline Newportopora & undulata & 96.0 & 453.0 & 457.0 & 445.0 \\
\hline Nicholsonella & ponderosa & 48.7 & 453.0 & 465.0 & 445.0 \\
\hline Dybowskites & laxatus & 7.6 & 453.5 & 458.1 & 451.7 \\
\hline Nolvakites & clavus & 7.0 & 453.5 & 454.0 & 453.0 \\
\hline Prasopora & grayae & 26.2 & 453.7 & 458.4 & 443.8 \\
\hline Halloporina & crenulata & 22.6 & 454.0 & 456.0 & 450.0 \\
\hline Hemiphragma & instabilis & 147.8 & 454.0 & 465.0 & 445.2 \\
\hline Homotrypella & aequalis & 107.7 & 454.0 & 457.0 & 445.2 \\
\hline Monotrypella & irrasum & 18.1 & 454.0 & 457.0 & 450.0 \\
\hline Tarphophragma & multitabulata & 15.6 & 454.0 & 465.0 & 445.0 \\
\hline Bimuropora & dubia & 15.2 & 454.5 & 465.0 & 445.0 \\
\hline Atactopora & typicalis & 19.2 & 455.0 & 458.4 & 443.8 \\
\hline Atactoporella & hirsuta & 6.5 & 455.0 & 457.0 & 442.0 \\
\hline Diazipora & monotrypoides & 4.5 & 455.5 & 467.3 & 452.0 \\
\hline Goldfussitrypa & esthoniae & 106.1 & 455.5 & 458.4 & 449.0 \\
\hline Mesotrypina & milleporacea & 126.7 & 455.5 & 457.0 & 454.0 \\
\hline Acantholaminatus & typicus & 11.9 & 456.0 & 457.0 & 445.0 \\
\hline Aostipora & sublamellosa & 24.5 & 457.5 & 470.0 & 445.2 \\
\hline Capillapora & arcuata & 11.8 & 457.5 & 458.0 & 457.0 \\
\hline Orbignyella & maculatum & 4.5 & 457.5 & 456.0 & 453.0 \\
\hline Phragmopora & cystata & 18.5 & 457.5 & 470.0 & 443.8 \\
\hline Phragmoporella & multiporarum & 17.8 & 457.5 & 463.0 & 456.0 \\
\hline Diplotrypa & petropolitana & 140.0 & 460.9 & 467.3 & 443.8 \\
\hline Jordanopora & heroensis & 110.7 & 461.0 & 465.0 & 456.0 \\
\hline Lamottopora & duncanae & 96.2 & 461.0 & 465.0 & 456.0 \\
\hline Champlainopora & chazyensis & 34.1 & 461.2 & 460.0 & 453.0 \\
\hline Rozhnovites & limatus & 19.9 & 462.8 & 470.3 & 464.5 \\
\hline Kanoshopora & droserae & 26.5 & 463.0 & 477.7 & 470.0 \\
\hline Trematoporina & intercludens & 77.0 & 464.2 & 470.0 & 423.0 \\
\hline Eichwaldipora & ovulum & 58.9 & 465.0 & 470.0 & 460.0 \\
\hline Dittopora & claevaeformis & 24.2 & 471.3 & 477.7 & 433.4 \\
\hline Orbiramus & normalis & 44.8 & 479.5 & 482.0 & 471.2 \\
\hline
\end{tabular}

Ma; Erwin 2006; Li et al. 2021). Both had major impacts on bryozoan faunas globally (Taylor and Larwood 1988; Tuckey and Anstey 1992; Powers and Bottjer 2009; Taylor 2020).
Increased originations did lead to greater trepostome diversity and thus more scatter in BSI values (Fig. 2). This is best seen 460-455 Ma during the Great Ordovician Biodiversification 
Table 2 Two sample $t$ test statistics for trepostome Bryozoan Skeletal Index (BSI) values across the transition from the Calcite I Sea to the Aragonite II Sea by zoarial habit

\begin{tabular}{|c|c|c|c|c|}
\hline & Age (Ma) of transition from Calcite I Sea to the Aragonite II Sea & 350 & 342 & 333 \\
\hline \multirow[t]{6}{*}{ All zoarial types } & Calcite I Sea mean BSI & 42.0 & 42.7 & 42.8 \\
\hline & Aragonite II Sea mean BSI & 46.9 & 45.6 & 45.8 \\
\hline & Degrees of freedom & 102 & 98 & 74 \\
\hline & t statistic & 0.804 & 0.476 & 0.446 \\
\hline & $P$ value & 0.424 & 0.635 & 0.657 \\
\hline & $\begin{array}{l}\text { Significant change from Calcite I Sea to the Aragonite } \\
\text { II Sea based on } P<0.05 \text { ? }\end{array}$ & No & No & No \\
\hline \multirow[t]{6}{*}{ Ramose zoaria } & Calcite I Sea mean BSI & 46.1 & 47.5 & 47.3 \\
\hline & Aragonite II Sea mean BSI & 50.5 & 47.3 & 47.9 \\
\hline & Degrees of freedom & 60 & 60 & 45 \\
\hline & t statistic & 0.541 & -0.028 & 0.064 \\
\hline & $P$ value & 0.591 & 0.978 & 0.949 \\
\hline & $\begin{array}{l}\text { Significant change from Calcite I Sea to the Aragonite } \\
\text { II Sea based on } P<0.05 \text { ? }\end{array}$ & No & No & No \\
\hline \multirow[t]{6}{*}{ Encrusting zoaria } & Calcite I Sea mean BSI & 22.9 & 22.8 & 23.7 \\
\hline & Aragonite II Sea mean BSI & 35.8 & 37.9 & 37.2 \\
\hline & Degrees of freedom & 26 & 20 & 17 \\
\hline & t statistic & 1.509 & 1.650 & 1.400 \\
\hline & $P$ value & 0.143 & 0.114 & 0.180 \\
\hline & $\begin{array}{l}\text { Significant change from Calcite I Sea to the Aragonite } \\
\text { II Sea based on } P<0.05 \text { ? }\end{array}$ & No & No & No \\
\hline \multirow[t]{6}{*}{ Massive zoaria } & Calcite I Sea mean BSI & 49.3 & 49.3 & 49.3 \\
\hline & Aragonite II Sea mean BSI & 56.9 & 56.9 & 56.9 \\
\hline & Degrees of freedom & 5 & 5 & 5 \\
\hline & t statistic & 0.285 & 0.285 & 0.285 \\
\hline & $P$ value & 0.787 & 0.787 & 0.787 \\
\hline & $\begin{array}{l}\text { Significant change from Calcite I Sea to the Aragonite } \\
\text { II Sea based on } P<0.05 \text { ? }\end{array}$ & No & No & No \\
\hline
\end{tabular}

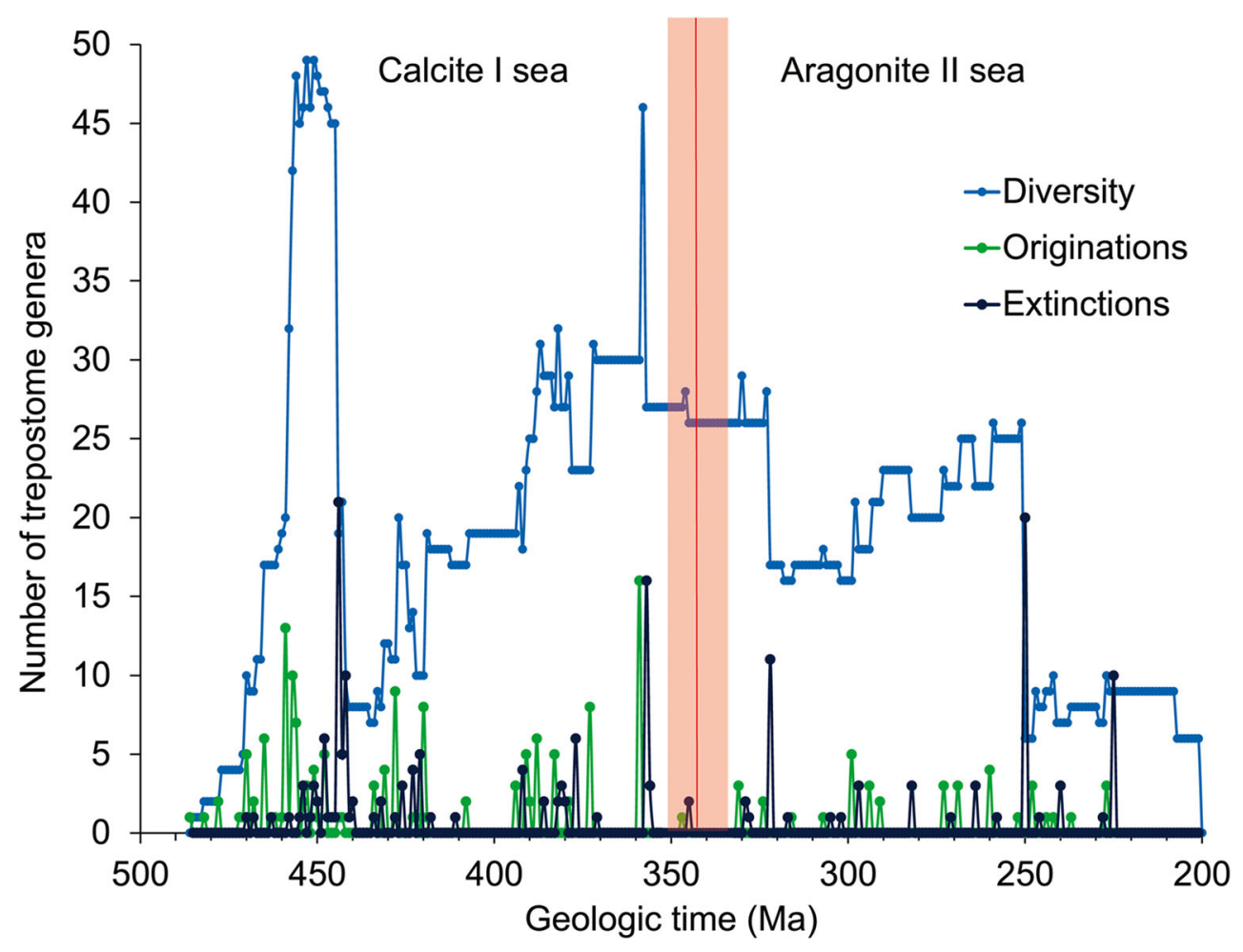

Fig. 3 Trepostome genus diversity and number of originations and extinctions over geologic time based on the stratigraphic range of each genus. Three different proposed ages for the transition from the Calcite I
Sea to the Aragonite II Sea are shown by the shaded red box. Left edge of red box is $350 \mathrm{Ma}$, vertical red line in centre is $342 \mathrm{Ma}$, and right edge of shaded box is $333 \mathrm{Ma}$ 
Event (GOBE) (Webby et al. 2004). The combination of increased diversity and BSI values may have contributed to bryozoans being the most diverse group of reef-building organisms during the GOBE (Ernst 2018; Servais and Harper 2018). The Ordovician diversification of trepostomes was a component of the GOBE (Taylor and Larwood 1990; Taylor and Ernst 2004), including as a substrate for the Ordovician Bioerosion Revolution (Mángano et al. 2016).

The lack of change from the Calcite I Sea into the Aragonite II Sea suggests that the trepostomes were accommodating the change in seawater chemistry in some other way. Laboratory experiments on extant passive aragonite and calcite biomineralisers grown in the different seawater $\mathrm{Mg} / \mathrm{Ca}$ ratios (Ries 2005; Stanley et al. 2010; Mewes et al. 2014) suggest that passive biomineralisers respond to unfavourable seawater conditions by changing skeletal composition. Thus, the calcitic trepostomes would have been using more energy to precipitate their skeletons in the less favourable seawater chemistry in the Aragonite II Sea, so they may have actively channelled more resources to calcification. Alternatively, they may have been dealing with the changing ocean chemistry metabolically or reproductively, not structurally as evidenced in the BSI. The fact that there was no change in BSI values, diversity, originations, or extinctions at the Calcite I Sea transition into the Aragonite II Sea provides strong evidence that trepostomes were not passive hyper-calcifiers but actively managed their calcification.

From a mineralogy perspective, modern bryozoans are not passive biomineralisers. They can precipitate a mix of LMC, $\mathrm{HMC}$, and aragonite where needed in their skeletons tailored to the functional needs of the colonies (Smith et al. 2006; Taylor et al. 2009). Thus, they are best described as active biomineralisers. This has been previously argued for the postPalaeozoic cheilostomes (Smith et al. 2006; Taylor et al. 2009) and the Palaeozoic trepostomes (Taylor and Kuklinski 2011).

In Taylor and Kuklinski's (2011) study, they used two independent proxies (i.e. branch diameter and ZWT) for degree of calcification in trepostomes. We used BSI which incorporates three parameters including exozone width instead of branch diameter which is composed of the highly calcified exozone and the minimally calcified endozone. They looked only at dendroid (i.e. ramose) forms, whereas we included all zoarial habits. They compiled their data from the literature for 188 species in 44 genera from the Ordovician (calcite sea), Devonian (calcite sea), and Permian (aragonite sea), whereas we included all genera and the entire trepostome stratigraphic range from the Ordovician to the Triassic and all periods in between. Despite these differences in sample size and range, our results were similar. Trepostome calcification did not alter in response to changes in the $\mathrm{Mg} / \mathrm{Ca}$ ratio of seawater. Factors other than ocean chemistry control their calcification. Trepostomes, and likely all bryozoans, are active biomineralisers, not passive hypercalcifiers.
Acknowledgements Robert S. Nelson, III, collected some of this data as part of his senior thesis at Dickinson College. Caroline Buttler provided access to the genus descriptions and plates for the forthcoming Trepostome volume of the Treatise. This manuscript was greatly improved by the thoughtful reviews of Andrej Ernst and Abigail Smith.

Funding Open Access funding provided by the IReL Consortium.

\section{Declarations}

Conflict of interest The authors declare that they have no conflict of interest.

Open Access This article is licensed under a Creative Commons Attribution 4.0 International License, which permits use, sharing, adaptation, distribution and reproduction in any medium or format, as long as you give appropriate credit to the original author(s) and the source, provide a link to the Creative Commons licence, and indicate if changes were made. The images or other third party material in this article are included in the article's Creative Commons licence, unless indicated otherwise in a credit line to the material. If material is not included in the article's Creative Commons licence and your intended use is not permitted by statutory regulation or exceeds the permitted use, you will need to obtain permission directly from the copyright holder. To view a copy of this licence, visit http://creativecommons.org/licenses/by/4.0/.

\section{References}

Balthasar, U., \& Cusack, M. (2015). Aragonite-calcite seas-Quantifying the gray area. Geology, 43, 99-102. https://doi.org/10.1130/ G36293.1.

Bond, D. P. G., \& Gasby, S. E. (2020). Late Ordovician mass extinction caused by volcanism, warming, and anoxia, not cooling and glaciation. Geology, 48, 777-781. https://doi.org/10.1130/G47377.1.

Burton, E. A., \& Walter, L. M. (1991). The effects of $\mathrm{PCO}_{2}$ and temperature on magnesium incorporation in calcite in seawater and $\mathrm{MgCl}_{2}$ $\mathrm{CaCl}_{2}$ solutions. Geochimica et Cosmochimica Acta, 55, 777-785. https://doi.org/10.1016/0016-7037(91)90341-2.

Cohen, K. M., Harper, D. A. T., Gibbard, P. L., \& Fan, J.-X. (2020). The ICS international chronostratigraphic chart (version 2020/03). International Commission on Stratigraphy. Retrieved February 14 2021 from https://stratigraphy.org/ICSchart/ChronostratChart202003.pdf

Dickson, J. A. D. (2002). Fossil echinoderms as monitor of the $\mathrm{Mg} / \mathrm{Ca}$ ratio of Phanerozoic oceans. Science, 298, 1222-1224. https://doi. org/10.1306/112203740355.

Dickson, J. A. D. (2004). Echinoderm skeletal preservation: Calcitearagonite seas and the $\mathrm{Mg} / \mathrm{Ca}$ ratio of Phanerozoic oceans. Journal of Sedimentary Research, 74, 355-365. https://doi.org/10.1306/ 112203740355.

Dijk, I. van, de Nooijer, L. J., Hart, M. B., \& Reichart, G.-J. (2016). The long-term impact of magnesium in seawater on foraminiferal mineralogy: Mechanism and consequences. Global Biogeochemical Cycles, 30, 438-446. https://doi.org/10.1002/2015GB005241.

Ernst, A. (2018). Diversity dynamics of Ordovician bryozoa. Lethaia, 51, 198-206. https://doi.org/10.1111/let.12235.

Ernst, A. (2020). 2. Fossil record and evolution of Bryozoa. In T. Schwaha (Ed.), Phylum Bryozoa (pp. 11-55). De Gruyter. https:// doi.org/10.1515/9783110586312-002. 
Erwin, D. H. (2006). Extinction: How life on earth nearly ended 250 million years ago. Princeton University Press ISBN 0-691-00524-9.

Farkaš, J., Böhm, F., Wallmann, K., Blenkinsop, J., Eisenhauer, A., van Geldern, R., Munnecke, A., Voigt, S., \& Veizer, J. (2007). Calcium isotope record of Phanerozoic oceans: Implications for chemical evolution of seawater and its causative mechanisms. Geochimica et Cosmochimica Acta, 71, 5117-5134. https://doi.org/10.1016/j. gca.2007.09.004.

Hardie, L. A. (1996). Secular variation in seawater chemistry: An explanation for the coupled secular variation in the mineralogies of marine limestones and potash evaporites over the past 600 m.y. Geology, 24, 279-283. https://doi.org/10.1130/0091-7613(1996) 024<0279:SVISCA >2.3.CO;2.

ICZN. (1999). International code of zoological nomenclature. International Commission on Zoological Nomenclature ISBN 085301-006-4.

Key Jr., M. M., Wyse Jackson, P. N., \& Felton, S. H. (2016). Intracolony variation in colony morphology in reassembled fossil ramose stenolaemate bryozoans from the Upper Ordovician (Katian) of the Cincinnati Arch region, USA. Journal of Paleontology, 90, 400-412. https://doi.org/10.1017/jpa.2016.66.

Kiessling, W., Aberhan, M., \& Villier, L. (2008). Phanerozoic trends in skeletal mineralogy driven by mass extinctions. Nature Geoscience, 1, 527-530. https://doi.org/10.1038/ngeo251.

Li, M., Grasby, S. E., Wang, S.-J., Zhang, X., Wasylenki, L. E., Xu, Y., Sun, M., Beauchamp, B., Hu, D., \& Shen, Y. (2021). Nickel isotopes link Siberian traps aerosol particles to the end-Permian mass extinction. Nature Communications, 12, 2024. https://doi.org/10. 1038/s41467-021-22066-7.

Lowenstein, T. K., Timofeeff, M. N., Brennan, S. T., Hardie, L. A., \& Demicco, R. V. (2001). Oscillations in Phanerozoic seawater chemistry: Evidence from fluid inclusions. Science, 294, 1086-1088. https://doi.org/10.1126/science.1064280.

Mackenzie, F. T., \& Pigott, J. D. (1981). Tectonic controls of Phanerozoic sedimentary rock cycling. Journal of the Geological Society, 138, 183-196. https://doi.org/10.1144/gsjgs.138.2.0183.

Mángano, M. G., Buatois, L. A., Wilson, M., \& Droser, M. (2016). The great Ordovician biodiversification event. In M. G. Mángano \& L. A. Buatoi (Eds.), The trace-fossil record of major evolutionary events (pp. 127-156). Springer, Topics in Geobiology 39. https:// doi.org/10.1007/978-94-017-9600-2_4.

Mewes, A., Langer, G., Jan de Nooijer, L., Bijma, J., \& Reichart, G.-J. (2014). Effect of different seawater $\mathrm{Mg}^{2+}$ concentrations on calcification in two benthic foraminifers. Marine Micropaleontology, 113, 56-64. https://doi.org/10.1016/j.marmicro.2014.09.003.

Montañez, I. P. (2002). Biological skeletal carbonate records changes in major-ion chemistry of paleo-oceans. Proceedings of the National Academy of Sciences, 99, 15,852-15,854. https://doi.org/10.1073/ pnas.262659599.

Palmer, A. R. (1983). The decade of North American geology 1983 geologic time scale. Geology, 11, 503-504. https://doi.org/10. 1130/0091-7613(1983)11<503:TDONAG>2.0.CO;2.

Porter, S. M. (2010). Calcite and aragonite seas and the de novo acquisition of carbonate skeletons. Geobiology, 8, 256-277. https://doi.org/ 10.1111/j.1472-4669.2010.00246.x.

Powers, C. M., \& Bottjer, D. J. (2009). Behavior of lophophorates during the end-Permian mass extinction and recovery. Journal of Asian Earth Sciences, 36, 413-419. https://doi.org/10.1016/j.jseaes.2008. 05.002 .

Quattrini, A. M., Rodríguez, E., Faircloth, B. C., Cowman, P. F., Brugler, M. R., Farfan, G. A., Hellberg, M. E., Kitahara, M. V., Morrison, C. L., Paz-García, D. A., Reimer, J. D., \& McFadden, C. S. (2020). Palaeoclimate ocean conditions shaped the evolution of corals and their skeletons through deep time. Nature Ecology \& Evolution, 4, 1531-1538. https://doi.org/10.1038/s41559-020-01291-1.
Ries, J. B. (2004). Effect of ambient $\mathrm{Mg} / \mathrm{Ca}$ ratio on $\mathrm{Mg}$ fractionation in calcareous marine invertebrates: A record of the oceanic $\mathrm{Mg} / \mathrm{Ca}$ ratio over the Phanerozoic. Geology, 32, 981-984. https://doi.org/ 10.1130/G20851.1.

Ries, J. B. (2005). Aragonite production in calcite seas: Effect of seawater $\mathrm{Mg} / \mathrm{Ca}$ ratio on calcification and growth of the calcareous alga Penicillus capitatus. Paleobiology, 31, 445-458. https://doi.org/10. 1666/0094-8373(2005)031[0445:APICSE]2.0.CO;2.

Ries, J. B. (2010). Review: Geological and experimental evidence for secular variation in seawater $\mathrm{Mg} / \mathrm{Ca}$ (calcite-aragonite seas) and its effects on marine biological calcification. Biogeosciences, 7, 27952849. https://doi.org/10.5194/bg-7-2795-2010.

Sandberg, P. A. (1975). New interpretations of Great Salt Lake ooids and of ancient nonskeletal carbonate mineralogy. Sedimentology, 22, 497-537. https://doi.org/10.1111/j.1365-3091.1975.tb00244.x.

Sandberg, P. A. (1983). An oscillating trend in Phanerozoic non-skeletal carbonate mineralogy. Nature, 305, 19-22. https://doi.org/10.1038/ $305019 \mathrm{a} 0$.

Schlager, W. (2005). Secular oscillations in the stratigraphic record - an acute debate. Facies, 51, 13-17. https://doi.org/10.1007/s10347005-0066-5.

Servais, T., \& Harper, D. A. T. (2018). The great Ordovician biodiversification event (GOBE): Definition, concept and duration. Lethaia, 51, 151-164. https://doi.org/10.1111/let.12259.

Sheehan, P. M. (2001). The Late Ordovician mass extinction. Annual Review of Earth and Planetary Sciences, 29, 331-364. https://doi. org/10.1146/annurev.earth.29.1.331.

Siemann, M. G. (2003). Extensive and rapid changes in seawater chemistry during the Phanerozoic: Evidence from $\mathrm{Br}$ contents in basal halite. Terra Nova, 15, 243-248. https://doi.org/10.1046/j.13653121.2003.00490.x.

Smith, A. M., Nelson, C. S., \& Spencer, H. G. (1998). Skeletal carbonate mineralogy of New Zealand bryozoans. Marine Geology, 151, 27 46. https://doi.org/10.1016/S0025-3227(98)00055-3.

Smith, A. M., Key Jr., M. M., \& Gordon, D. P. (2006). Skeletal mineralogy of bryozoans: Taxonomic and temporal patterns. Earth Science Reviews, 78, 287-306. https://doi.org/10.1016/j.earscirev. 2006.06.001.

Stanley, S. M. (2006). Influence of seawater chemistry on biomineralization throughout Phanerozoic time: Paleontological and experimental evidence. Palaeogeography, Palaeoclimatology, Palaeoecology, 232, 214-236. https://doi.org/10.1016/j.palaeo.2005.12.010.

Stanley, S. M., \& Hardie, L. A. (1998). Secular oscillations in the carbonate mineralogy of reef-building and sediment-producing organisms driven by tectonically forced shifts in seawater chemistry. Palaeogeography, Palaeoclimatology, Palaeoecology, 144, 3-19. https://doi.org/10.1016/S0031-0182(98)00109-6.

Stanley, S. M., \& Hardie, L. A. (1999). Hypercalcification: Paleontology links plate tectonics and geochemistry to sedimentology. GSA Today, 9, 1-7.

Stanley, S. M., Ries, J. B., \& Hardie, L. A. (2002). Low-magnesium calcite produced by coralline algae in seawater of Late Cretaceous composition. Proceedings of the National Academy of Sciences, 99, 15323-15326. https://doi.org/10.1073/pnas.232569499.

Stanley, S. M., Ries, J. B., \& Hardie, L. A. (2005). Seawater chemistry, coccolithophore population growth, and the origin of Cretaceous chalk. Geology, 33, 593-596. https://doi.org/10.1130/G21405.1.

Stanley, S. M., Ries, J. B., \& Hardie, L. A. (2010). Increased production of calcite and slower growth for the major sediment-producing alga Halimeda as the $\mathrm{Mg} / \mathrm{Ca}$ ratio of seawater is lowered to a "calcite sea" level. Journal of Sedimentary Research, 80, 6-16. https://doi. org/10.2110/jsr.2010.011.

Steuber, T., \& Veizer, J. (2002). Phanerozoic record of plate tectonic control of seawater chemistry and carbonate sedimentation. Geology, 30, 1123-1126. https://doi.org/10.1130/0091-7613(2002) 030<1123:PROPTC $>2.0 . \mathrm{CO} ; 2$. 
Tavener-Smith, R., \& Williams, A. (1972). The secretion and structure of the skeleton of living and fossil Bryozoa. Philosophical Transactions of the Royal Society of London, B, Biological Sciences, 264, 97-159. https://doi.org/10.1098/rstb.1972.0010.

Taylor PD (1993) Bryozoa. In. M. J. Benton (Ed.), The fossil record 2 (pp. 465-489). Chapman and Hall.

Taylor, P. D. (2020). Bryozoan paleobiology. Wiley Blackwell.

Taylor, P. D., \& Allison, P. A. (1998). Bryozoan carbonates through time and space. Geology, 26, 459-462. https://doi.org/10.1130/00917613(1998)026<0459:BCTTAS >2.3.CO;2.

Taylor, P. D., \& Ernst, A. (2004). Bryozoan diversification during the Ordovician. In B. D. Webby, M. L. Droser, \& F. Paris (Eds.), The Great Ordovician Biodiversification Event (pp. 147-156). Columbia University Press.

Taylor, P. D., \& Kuklinski, P. (2011). Seawater chemistry and biomineralization: Did trepostome bryozoans become hypercalcified in the 'calcite sea' of the Ordovician? Palaeobiodiversity and Palaeoenvironments, 91(3), 185-195. https://doi.org/10.1007/s12549-011-0054-4.

Taylor, P. D., \& Larwood, G. P. (1988). Mass extinctions and the pattern of bryozoan evolution. In G. P. Larwood (Ed.), Extinction and Survival in the Fossil Record (pp. 99-119). Systematics Association.

Taylor, P. D., \& Larwood, G. P. (1990). Major evolutionary radiations in the Bryozoa. In P. D. Taylor \& G. P. Larwood (Eds.), Major Evolutionary Radiations (pp. 209-233). Systematics Association.

Taylor, P. D., \& Wilson, M. A. (1999). Dianulites Eichwald, 1829: An unusual Ordovician bryozoan with a high-magnesium calcite skeleton. Journal of Paleontology, 73, 38-48. https://doi.org/10.1017/ S0022336000027529.

Taylor, P. D., James, N. P., Bone, Y., Kuklinski, P., \& Kyser, T. K. (2009). Evolving mineralogy of cheilostome bryozoans. Palaios, 24, 440-452. https://doi.org/10.2110/palo.2008.p08-124r.
Taylor, P. D., Vinn, O., \& Wilson, M. A. (2010). Evolution of biomineralization in lophophorates. Special Papers in Palaeontology, 84, 317-333. https://doi.org/10.1111/j.1475-4983.2010.00985.x.

Tuckey, M. E., \& Anstey, R. L. (1992). Late Ordovician extinctions of bryozoans. Lethaia, 25, 111-117. https://doi.org/10.1111/j.15023931.1992.tb01795.x.

Turchyn, A. V., \& DePaolo, D. J. (2019). Seawater chemistry through Phanerozoic time. Annual Review of Earth and Planetary Sciences, 47, 197-224. https://doi.org/10.1146/annurev-earth-082517010305.

Webb, G. E., \& Sorauf, J. E. (2002). Zigzag microstructure in rugose corals: A possible indicator of relative seawater $\mathrm{Mg} / \mathrm{Ca}$ ratios. Geology, 30, 415-418. https://doi.org/10.1130/0091-7613(2002) 030<0415:ZMIRCA $>2.0 . \mathrm{CO} ; 2$.

Webby, B. D., Droser, M. L., Paris, F., \& Percival, I. G. (2004). The great Ordovician biodiversification event. Columbia University Press.

Wilkinson, B. H., \& Givens, K. R. (1986). Secular variation in abiotic marine carbonates: Constraints on Phanerozoic atmospheric carbon dioxide contents and oceanic $\mathrm{Mg} / \mathrm{Ca}$ ratios. Journal of Geology, 94, 321-333. https://doi.org/10.1086/629032.

Wyse Jackson, P. N., Key Jr., M. M., \& Reid, C. M. (2020). Bryozoan skeletal index (BSI): A measure of the degree of calcification in stenolaemate bryozoans. In P. N. Wyse Jackson \& K. Zagorsek (Eds.), Bryozoan Studies 2019 (pp. 193-206). Czech Geological Survey.

Zhuravlev, A. Y., \& Wood, R. A. (2009). Controls on carbonate skeletal mineralogy: Global $\mathrm{CO}_{2}$ evolution and mass extinctions. Geology, 37, 1123-1126. https://doi.org/10.1130/G30204A.1.

Publisher's note Springer Nature remains neutral with regard to jurisdictional claims in published maps and institutional affiliations. 